\title{
BMJ Open The effect of enhanced public-private partnerships on Maternal, Newborn and child Health Services and outcomes in Nairobi-Kenya: the PAMANECH quasi-experimental research protocol
}

\author{
Pauline Bakibinga, Remare Ettarh, Abdhalah K Ziraba, Catherine Kyobutungi, \\ Eva Kamande, Nicholas Ngomi, Jane Osindo
}

To cite: Bakibinga $P$, Ettarh R, Ziraba AK, et al. The effect of enhanced publicprivate partnerships on Maternal, Newborn and child Health Services and outcomes in Nairobi-Kenya: the PAMANECH quasi-experimental research protocol. BMJ Open 2014;4: e006608. doi:10.1136/ bmjopen-2014-006608

- Prepublication history for this paper is available online. To view these files please visit the journal online (http://dx.doi.org/10.1136/ bmjopen-2014-006608).

Accepted 30 September 2014

CrossMark

Health Challenges and Systems Research Program, African Population \& Health Research Center, Nairobi, Kenya

Correspondence to Dr P Bakibinga; paulabak80@gmail.com

\section{ABSTRACT}

Introduction: Rapid urbanisation in Kenya has resulted in growth of slums in urban centres, characterised by poverty, inadequate social services and poor health outcomes. The government's initiatives to improve access to quality healthcare for mothers and children are largely limited to public health facilities, which are few and/or inaccessible in underserved areas such as the slums. The 'Partnership for Maternal, Newborn and Child Health' (PAMANECH) project is being implemented in two Nairobi slums, Viwandani and Korogocho, to assess the impact of strengthening public-private partnerships for the delivery of healthcare on the health of mothers, newborns and young children in two informal settlements in Kenya.

Methods and analysis: This is a quasi-experimental study; our approach is to support private as well as public health providers and the community to enhance access to and demand for quality healthcare services. Key activities include: infrastructural upgrade of selected Private Not-For-Profit health facilities operating in the two slums, building capacity for healthcare providers as well as the Health Management Teams in Nairobi, facilitating provision of supportive supervision by the local health authorities and forming networks of Community Health Volunteers (CHVs) to create demand for health services. To assess the impact of the intervention, the study is utilising multiple data sources using a combination of qualitative and quantitative methods. A baseline survey was conducted in 2013 and an end-line survey will be conducted at least 1 year after full implementation of the intervention. Systematic monitoring and documentation of the intervention is ongoing to strengthen the case for causal inference.

Ethics and dissemination: Ethical approval for the study was obtained from the Kenya Medical Research Institute. Key messages from the results will be packaged and widely disseminated through workshops, conference presentations, reports, factsheets and academic publications to facilitate uptake by policymakers.

Protocol registration number: KEMRI- NON-SSCPROTOCOL No. 393.

\section{Strengths and limitations of this study}

The project seeks to address most challenges in the healthcare system in a slum context: infrastructural, human resource, information, financing, technologies and supplies.

- It demonstrates in action that intervention based on research evidence can change the health outcomes of a community.

- Compliance by all actors is very important for the success of this project.

\section{BACKGROUND}

Like many other sub-Saharan African countries, Kenya is experiencing rapid urbanisation growth characterised by matching expansion of informal settlements. ${ }^{1}{ }^{2}$ Slums and slum-like settlements are home to about $60 \%$ of urban residents in Kenya. ${ }^{3}$ However, the public healthcare delivery system has not kept pace with urban population growth, being neither accessible nor responsive to the critical health needs of slum residents. ${ }^{45}$ This is, in part, due to the fact that the existing public health facilities were only intended to serve the formal settlements and not the large concentrations of people within informal settlements. In addition, the informal nature of slums and, by implication, the uncertainty about the legal status of the settlements has for a long time made the improvement of public social services in these areas difficult. ${ }^{6}$ The Kenyan government's commitment to improve access to quality healthcare for mothers and children is demonstrated by such initiatives as free access to maternity care available at public health facilities. However, public health facilities are almost non-existent and inaccessible to most mothers and their children in 
underserved areas such as in the urban informal settlements. ${ }^{5}$

Research conducted in two slums of Nairobi (Korogocho and Viwandani) over the past decade has documented the near absence of public health facilities in these settlements and the health consequences of this. ${ }^{578}$ Korogocho slum is served by two public health facilities while Viwandani is served by one, all located on the outskirts of the slums. In 2008-2009, a service provision assessment conducted by the African Population and Health Research Center (APHRC) in these and one other slum showed that less than $1 \%$ of health facilities serving these settlements were public. ${ }^{9}$ The gap left by the near absence of the public sector has been filled by a vibrant private health sector, which is largely unregulated and unsupported. Although private providers cater for a significant proportion of slum residents, healthcare workers in this sector rarely benefit from in-service training to update their skills to offer essential services such as basic and comprehensive emergency and obstetric care, family planning and management of common childhood illnesses. The linkages between the healthcare delivery system and other health-producing systems are also weak. In addition, costly health services with a high societal benefit, such as vaccination and family planning are usually provided at public facilities, which are largely inaccessible to slum residents.

The Kenyan Public-Private Partnership Bill 2011 and its policy framework opened up the provision of public services for private capital and expertise, creating a favourable environment for private sector participation in the provision of public services. ${ }^{10}$ The government of Kenya has put in place mechanisms through which private providers can meaningfully participate in the planning, monitoring and provision of health services at different levels through so-called health stakeholder forums. Private providers who are part of a District Health Stakeholders Forum are eligible to receive support in the form of essential supplies such as vaccines, tuberculosis drugs, family planning commodities and drugs for treating sexually transmitted infections. This support is contingent on meeting other quality standards and criteria such as staffing, infrastructure and the submission of timely reports from the routine Health Management Information System (HMIS). The $\mathrm{WHO}^{11}$ (figure 1) has described the building blocks of a health system that, when working as a whole to produce services that are accessible, reaching most people, of good quality and with the right safeguards, will lead to a well-functioning system that will produce good health for the people it is intended to serve.

Against this background, drawing on the aforementioned government initiatives and the WHO framework to improve private provider health services, and on the basis of the evidence generated from over a decade of research in the two slums, APHRC decided to design, implement and evaluate a package of interventions aimed at improving healthcare delivery. The Partnership

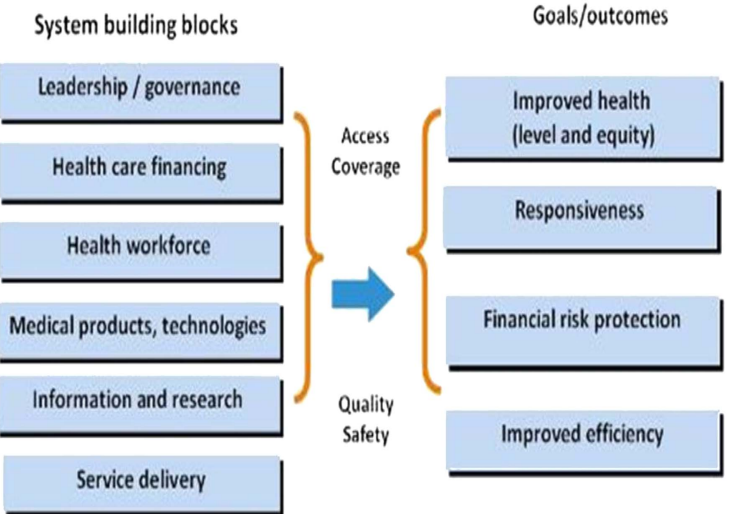

for Maternal, Newborn and Child Health (PAMANECH) project was designed and rolled out in July 2012 by the APHRC in Korogocho and Viwandani with a broader aim of strengthening the healthcare delivery system in urban informal settlements to be more responsive to the healthcare needs of mothers and their children.

The proposed work is grounded in classical systems thinking but within the WHO systems strengthening framework (figure 2). ${ }^{11}$ Classical systems' thinking acknowledges that 'problems' are part of the system and..."the component parts of a system can best be understood in the context of relationships with each other and with other systems, rather than in isolation..." ${ }^{11}$ The WHO health systems framework describes six building blocks that may be viewed as inputs and processes aimed at delivering outputs, outcomes and impact (figure 1). The effect of the inputs and processes may be assessed at the system level or by examining results in the population the system is intended to serve. Extending this framework to classical systems thinking (figure 2) recognises that the health system's 'problems' or its outputs, outcomes and impact are not only influenced by the system, but they may shape the kind of system that exists. For example, the high levels of morbidity, mortality and low utilisation of preventive services among slum residents are a result of inadequate inputs and processes in the public and private health system that serves this population. Other systems or factors may influence inputs, processes, outputs and outcomes at the system and population level. For instance, insecurity in the slums and poor social infrastructure affect the ability of private providers to attract staff and impede the population's ability to access health services. Other factors, including poor water and sanitation infrastructure, and food insecurity have a direct effect on health outcomes. The PAMANECH initiative seeks to directly address the following critical elements of the system: (1) service delivery; (2) leadership and governance; (3) health workforce; and (4) health information system. The initiative will also indirectly address the elements of healthcare financing, medical products, vaccines and technologies through strengthening leadership and management of healthcare services. 
Figure 2 Classical systems thinking applied to the WHO health system strengthening framework.

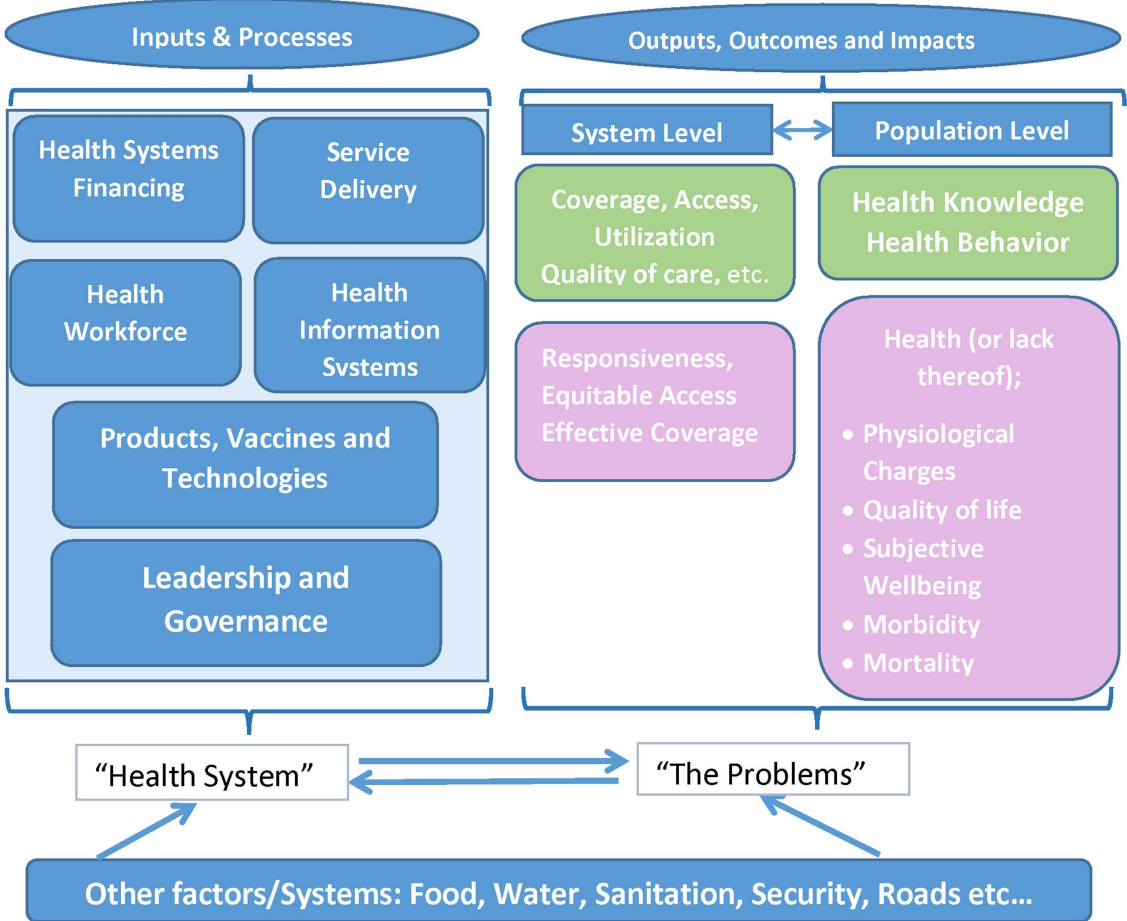

\section{PROJECT DESIGN AND LEARNING QUESTION}

The project utilises a quasi-experimental design. The main learning question is: 'Does strengthening the private sector with support from the public sector for the delivery of quality health services for slum residents have an impact of Maternal, Newborn and Child Health (MNCH) outcomes?'

\section{Specific learning questions}

The specific learning questions of primary interest are:

1. What effect has material support to private providers had on quality of care?

2. What is the effect of training in service guidelines on provider skills for the provision of MNCH services?

3. What is the effect of a network of CHVs on uptake of MNCH services in slum settlements?

4. What is the cost-effectiveness of the proposed intervention?

In addressing these questions, we will try to understand how or the ways through which a public-private partnership can contribute to service delivery to disadvantaged populations and the impact of such a partnership on MNCH indicators.

\section{METHODS AND ANALYSIS \\ Project setting}

The intervention site covers two informal settlements in Nairobi, namely Korogocho and Viwandani, where APHRC has been operating the Nairobi Urban Health and Demographic Surveillance System (NUHDSS) since 2003. The NUHDSS has been described in detail elsewhere and for this intervention and corresponding planned evaluation provides a reliable sampling frame. ${ }^{12}$
Viwandani and Korogocho settlements are together home to approximately 70000 residents and, like other slums, are characterised by poverty, poor coverage of social services and poor MNCH outcomes. More than a decade of research by APHRC in Viwandani and Korogocho slums shows that the two areas exhibit poor child, neonatal and maternal health indicators including high levels of maternal mortality (706/100000 compared to $488 / 100000$, the national average), high infant and under-five mortality. ${ }^{8}{ }^{13}$ In addition, stillbirth rates are unacceptably high. ${ }^{8}$ Whereas the proportion of births taking place in health facilities has increased significantly over the last decade $(52 \%$ in 2000 to $81 \%$ in 2012), ${ }^{14} 15$ about $60 \%$ of health facilities in this setting lack trained staff and equipment to handle basic emergency obstetric and neonatal complications. ${ }^{4}$ All factors considered, the dismal state of maternal and child care services in this setting is a major contributor to the observed high maternal and under-five mortality observed in the slums of Nairobi. ${ }^{12}$

\section{Partners}

Implementation of the PAMANECH project is a joint collaboration among several partners including APHRC, the City County of Nairobi, sub-County Health Management Teams (Kasarani and Makadara subcounties), community leaders in Korogocho and Viwandani, private providers, youth leaders and Community Health Volunteers.

\section{Facility selection}

Six credible and established (not-for-profit) health facilities were selected in Korogocho and Viwandani (three 
in each site) for transformation into 'one-stop primary level centres' for preventive and curative MNCH services. The selection criteria were based on the National Health Insurance Fund (NHIF) Accreditation Manual ${ }^{16}$ for maternal health services at level 2 and enhanced with items from the SAFECARE manual. ${ }^{17}$ The NHIF accreditation manual is used in Kenya for accrediting health facilities at different levels for eligibility to receive NHIF refunds. SAFECARE is a continuous quality improvement tool to improve healthcare delivery in resource-restricted settings. It aims to support health providers and consistently improve quality to meet the healthcare needs of the population they serve.

The assessment criterion was reviewed by the sub-County Health Management Teams (sCHMTs) of Makadara and Kasarani to determine the relevance and completeness of the tool. The final assessment had two sections: an oral interview section, which covered the health facility details (ownership, relationship with sCHMT), registration of health facility, power supply, presence of qualified registered health personnel, and guidelines and procedures used in MNCH. The second section was an observation checklist, which covered the environment of the health facility, physical structure of the facility, water supply, equipment in consultation room, labour ward and laboratory, availability of a functional ambulance and availability of wards. Each item on the list was scored using a 5-point scale with the maximum score (best score) per item being 1 and minimum score (worst score) per item being 5 . A list with 53 health facilities was generated by members of the project management committees from the two communities and validated by the sCHMTs. Twenty two of the listed facilities were not assessed as they were stand-alone laboratories or pharmacies that offered treatment for minor ailments and occasionally offered delivery services. For each health facility, a sum of all the scores was generated and this was used to rank the 31 assessed facilities. The top three health facilities in each settlement were selected for support and the results communicated to the community leaders, sCHMTs and all assessed facility heads at a meeting organised by APHRC.

\section{Key project activities}

The activities below are aimed at improving access to quality healthcare and reducing the financial burden of health expenditure for the two slum communities in Nairobi mentioned in this study.

\section{Infrastructural upgrade of selected private not-for-profit} health facilities that already operate in the two slums

Six credible and established private not-for-profit (PNFP) providers were selected; however, due to financial constraints, only five have received infrastructural upgrade. Each selected facility will be supported to provide: (1) essential and basic obstetric care; (2) antenatal care; (3) maternity care; (4) postnatal care; (5) diagnosis and treatment of common childhood illnesses;
(6) vaccination; and (7) HIV/AIDS-related services such as Voluntary and Routine Counselling and Testing and elimination of mother-to-child transmission of HIV. The support to be provided is based on the identified needs of each facility and includes expansion/renovation of existing facilities, provision of basic equipment, and water and electricity connections, among others. The providers were selected in a consultative manner that involved all the relevant stakeholders. The support is being provided in a phased manner contingent on meeting set criteria for quality improvement. The rationale for this infrastructural support is based on: (1) the need to meet Ministry of Health standards and accreditation criteria, (2) the need for space to offer the envisaged expanded range of services and (3) the need to ensure long-term sustainability of the programme through linkages to other funding streams. Adequate infrastructure is a key accreditation criterion according to guidelines formulated by the NHIF. It is important that these facilities are accredited by the NHIF, not only to benefit from their health insurance scheme, but to benefit from other initiatives such as the output-based aid voucher scheme for reproductive health services currently being piloted in the two slums. There is also potential to benefit from other health insurance schemes such as one offered by Jamii Bora-one of the largest microfinance organisations targeting slum dwellers and street families.

\section{Capacity building for healthcare providers}

Clinical personnel of the selected facilities are being trained using the most appropriate and current clinical and practice guidelines for $\mathrm{MNCH}$. Training is conducted gradually to ensure that what is learnt is institutionalised and practiced before additional training is provided. The private health providers are to receive training and support to generate routine HMIS data and reports that will feed into the district monthly reports.

\section{Capacity building for health management teams}

The sub-County (Kasarani and Makadara) and County (Nairobi) health authorities are being facilitated to offer systematic supportive supervision to the 'One-Stop Primary Level Centres' to ensure that guidelines are adhered to, skills are reinforced and ultimately highquality services are offered. In addition, the health authorities are to ensure access to high-quality, low-cost medicine and supplies by the five health centres through existing channels, such as recommendation to the largest supplier of high quality low cost medicines and supplies to the PNFP sector in the country (Mission for Essential Drugs and Supplies-MEDS). Some members of the health management teams have been supported to receive training on supervision, leadership and management to enhance their capacity to effectively manage health services in the districts under which the two slums fall. 
Engagement of CHVs

Under the Ministry of Health community health strategy for improving health, CHVs have been identified in all villages in the country. These, with some facilitation, help implement public health activities including reporting on health events in the community. Owing to high attrition rates of these volunteers, we found it necessary to train more CHVs in order to reconstitute the community units. Ten CHVs per village were selected from the trained pool within the two slums (a total of 180 from 280 trained) to work with the private providers and to create linkages with the public sector. Their main roles include household monitoring for childhood illness and vaccination status, referrals to a primary healthcare facility (public or private), registration of pregnant women in the designated households, referrals for antenatal care and skilled attended delivery at the upgraded private health facilities, assisting mothers with birth planning and postnatal visits, and distribution of contraceptives. These activities, drawing on proven approaches in the utilisation of community health workers, will provide evidence to guide the implementation of the national community strategy.

\section{Engagement of youth groups}

Young men in each village, who are already in organised groups, have been mobilised to work as security escorts for women in need of urgent medical attention either for themselves or their young children at night. Insecurity at night is one of the reasons women deliver at home and why they delay seeking care when it is needed. We are piloting this context-specific security service organised and run by young people to determine the feasibility of the service, to set up the most appropriate mechanisms for contracting the youth groups, and to establish payment modalities, sustainability and best communication practices between the users and providers. Youth groups have been linked to CHVs with whom they liaise to get information on which mother or child requires their services.

\section{Establishment of ambulance services}

An emergency referral system is crucial in improving MNCH outcomes. This is especially so during hours when public transporters are no longer operating, for example, at night. On the other hand, at the time when public transport is available, it would be difficult for a non-emergency vehicle to navigate Nairobi's traffic jams whereas emergency vehicles are given priority. Referred patients can also obtain care (such as intravenous fluids, oxygen, injections) en route to the next level facility in a well-equipped ambulance as opposed to a private vehicle. An ambulance system will be set up building on existing ambulance services in the community. The ambulance will be used to transfer mothers and children who need referral to the nearest maternity hospital. In each of the target slum settlements, one ambulance will be provided by the programme to serve the network of private providers in the area. The ambulances will be centrally located and managed from two of the upgraded health facilities. Priority will be given to facilities or organisations that already run some form of ambulance service since they may be best placed to manage the service. Appropriate communication mechanisms will be put in place to ensure that the ambulance is accessible at all times whenever it is needed. Obstetric emergency transportation systems have been plagued by problems of non-sustainability either as a result of high cost or the rarity of emergency events in the face of continued inputs. ${ }^{18}$ It is recommended that such transport systems should be complemented by quality improvements in the primary referral system and the system should be open to other users to ensure sustainability. ${ }^{19}{ }^{20}$ To mitigate against the risk of non-sustainability we propose to diversify the client base for these ambulances to include non-obstetric and nonchild emergencies. Different cost structures will be put in place whereby obstetric and child emergencies will pay half the price and the others will pay full price.

\section{Establishment of outreach specialist services}

The project team will work with professional associations in Kenya, such as the Kenya Obstetrical and Gynaecological Society (KOGS) and Kenya Paediatric Association (KPA) to run outreach clinics for women and children in slum settlements. This programme will work with the private providers to set out defined schedules for specific services and to coordinate these with KOGS and KPA specialists. The outreach clinics will add value by offering services that may not be routinely provided at level 2 facilities such as treatment for sexually transmitted infections, cervical cancer screening, longterm family planning and breast cancer screening, among others.

\section{Support to other credible PNFP providers}

The above activities, though aimed at strengthening the service delivery system serving slum residents, may end up interfering in the healthcare market and squeezing out credible private providers not selected for support. To guard against this, we propose to create opportunities for other eligible but unselected providers to benefit from other means of support. These will include supportive supervision from the sCHMT, participation in training programmes, support to generate routine HMIS that enhances their chances to get essential supplies from the public sector, participation in monthly meetings between private providers in each district, which are organised by the sCHMT.

In figure 3, we summarise the intervention: aiming to improve the quality of services offered, improve accessibility to these services and improve efficiency in delivery of these services thereby enhancing sustainability. The proposed initiative will not create a new system, rather it will enhance the hitherto untapped synergies between the public and private sector in health service provision, 
Figure 3 Summary of the PAMANECH Intervention.

\begin{tabular}{|c|c|c|}
\hline Objective & Indicator & Data Sources \\
\hline $\begin{array}{l}\text { Improved health outcomes for } \\
\text { newborns }\end{array}$ & $\begin{array}{l}\text { Proportion of newborns initiating } \\
\text { breastfeeding within } 1 \text { hour of } \\
\text { birth } \\
\text {-Neonatal mortality rate }\end{array}$ & $\begin{array}{l}\text { HMIS } \\
\text {-NUHDSS } \\
\text {-KDHS }\end{array}$ \\
\hline $\begin{array}{l}\text { Improved health outcomes for } \\
\text { children }<5 \text { years }\end{array}$ & $\begin{array}{l}- \text {-Measles vaccination rate } \\
\text {-Full vaccination coverage (12- } \\
23 \text { months) } \\
\text {-Proportion of sick children }<5 \\
\text { years who seek care at a health } \\
\text { facility } \\
\text {-Under five mortality rate }\end{array}$ & $\begin{array}{l}\text {-HMIS } \\
\text {-NUHDSS } \\
\text {-KDHS }\end{array}$ \\
\hline $\begin{array}{l}\text { Improved health for women of } \\
\text { childbearing age }\end{array}$ & $\begin{array}{l}\text {-Contraceptive prevalence rate } \\
\text { - Proportion of unmet need for } \\
\text { family planning } \\
\text {-Proportion attending at least } 4 \\
\text { ANCs } \\
\text { - Proportion of deliveries assisted } \\
\text { by trained personnel }\end{array}$ & $\begin{array}{l}\text {-HMIS } \\
\text {-NUHDSS } \\
\text {-KDHS }\end{array}$ \\
\hline $\begin{array}{l}\text { Increased demand for health } \\
\text { services provided in the selected } \\
\text { facilities }\end{array}$ & $\begin{array}{l}\text {-\% increase in level of uptake of } \\
\text { health care services } \\
\text {-Proportion of women seeking } \\
\text { services from the facilities } \\
\text {-Proportion of women referred by } \\
\text { CHVs }\end{array}$ & $\begin{array}{l}- \text { CHV logs } \\
\text {-HMIS } \\
\text {-Ambulance logs }\end{array}$ \\
\hline $\begin{array}{l}\text { Home deliveries at night reduced } \\
\text { as a result of improved security }\end{array}$ & $\begin{array}{l}\text {-Percentage reduction in home } \\
\text { deliveries at night } \\
\text {-Proportion of obstetric night } \\
\text { admissions at the selected } \\
\text { facilities }\end{array}$ & $\begin{array}{l}- \text { HMIS } \\
- \text {-CHV logs } \\
\text {-Youth group logs }\end{array}$ \\
\hline $\begin{array}{l}\text { Improved quality of services } \\
\text { provided in the selected facilities }\end{array}$ & $\begin{array}{l}\text {-Proportion of women reporting } \\
\text { blood pressure measurements } \\
\text { taken during all ANC visits } \\
\text {-Proportion of women reporting } \\
\text { receiving injections to control } \\
\text { bleeding during the third stage of } \\
\text { labour. } \\
\text {-Number of women reporting to } \\
\text { have efficiently used the } \\
\text { ambulance services } \\
\text {-Proportion of women reporting } \\
\text { satisfaction with the quality of } \\
\text { service provided in the upgraded } \\
\text { facilities } \\
\text {-Number of supervisions/visits } \\
\text { reported by Health facilities }\end{array}$ & $\begin{array}{l}\text {-HMIS } \\
\text {-Visitors books } \\
\text {-Satisfaction surveys } \\
\text {-Qualitative assessments }\end{array}$ \\
\hline
\end{tabular}

strengthen existing governance structures, promote community involvement in health through the provision of critical non-health support and improve capacities to generate and utilise health information for planning, monitoring, evaluation and advocacy. As shown in figure 3 , we propose that a high-quality system, accessible and in demand, will result in improved $\mathrm{MNCH}$ outcomes.

\section{Intervention monitoring and evaluation}

The intervention has preintervention and postintervention project assessments for changes, if any, on the MNCH health services and population health outcomes. We conducted a baseline survey in August 2013 and plan to conduct an end-line at least 1 year after the full implementation of the intervention. In order to be able to strengthen the case for causal inference we are conducting systematic monitoring and documentation of the intervention based on our intervention (figure 3). The monitoring and documentation will also capture any other contextual factors that may influence the same outcomes as our intervention.

\section{Data collection and analysis plan}

The effects and impact of the programme will be determined by triangulating data (quantitative and qualitative) and information from different sources, examining trends where possible, and trying to find and support explanations for the observed findings (figure 4). Internationally recognised principles and standards for data collection and analysis will be followed.

Several data sources have been identified:

1. The Nairobi Urban Health and Demographic Surveillance System (NUHDSS): For secondary data analysis to assess preintervention, intervention and post-intervention trends of antenatal care, obstetric care (skilled attended delivery), immunisation coverage, neonatal mortality (including stillbirths) and childhood mortality. Under the NUHDSS, every four months, all households are visited and information on the status of members including migrations, births, deaths and healthcare use is ascertained. For deaths, a cause of death is ascertained using verbal autopsy.

2. Quantitative assessments: These will be carried out through household surveys of women in reproductive age and children aged under five years to determine 
Figure 4 PAMANECH Project

Data collection plan.
Partnership for maternal, newborn, and child health (PAMANECH) project

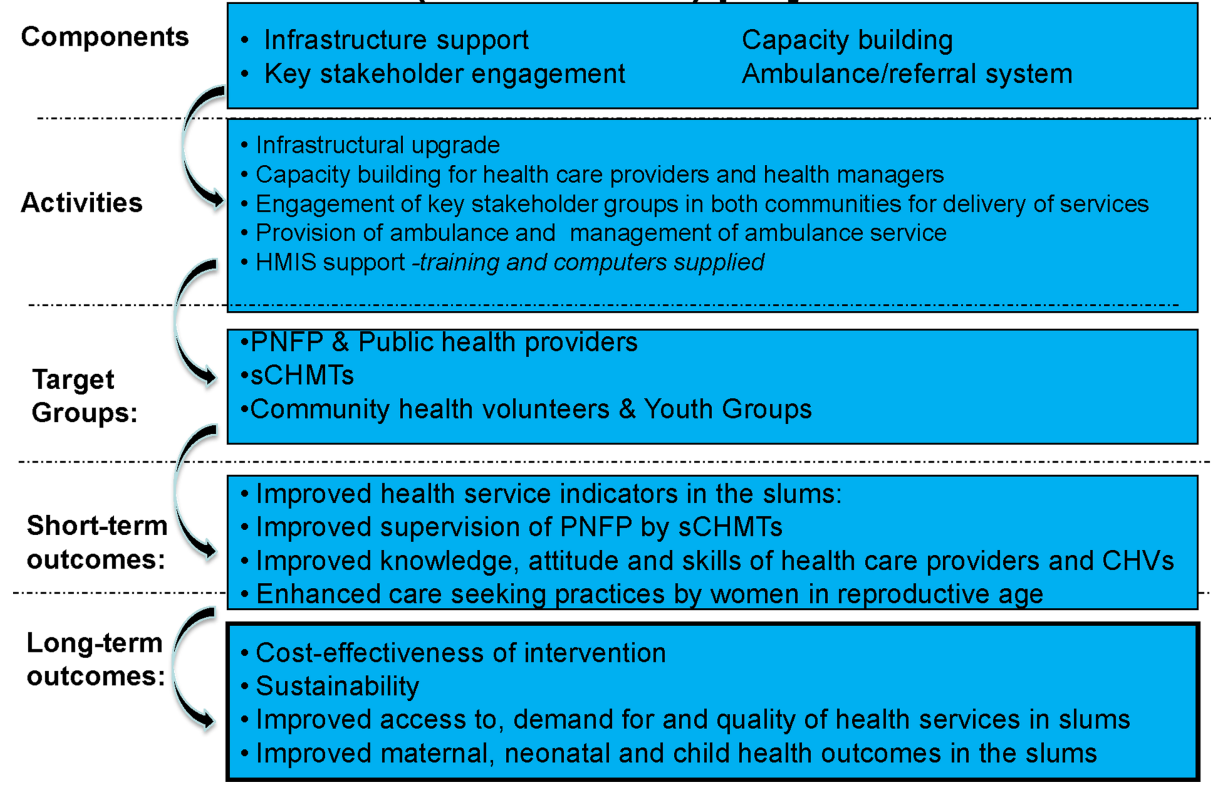

changes in healthcare utilisation patterns (if any) for preventive and curative services, interactions with Community Health Volunteers (CHVs), user satisfaction, healthcare costs and referral patterns. These data are not captured under the NUHDSS.

3. Routine HMIS: HMIS data from the selected and upgraded health facilities will be collated and quantitatively analysed to estimate attendance rates, case management practices against guidelines, supplies acquisition and uptake of different MNCH services, and referral patterns especially with regard to two-way referral by CHVs.

4. Qualitative assessments: These will be used to collect and analyse data from key informant interviews, in-depth interviews and focus group discussions with service providers and clients about the quality and accessibility of MNCH services, as well as the quality of interactions with CHVs.

5. Cost evaluation: This will entail a compilation of costs of goods and services from the provider perspective. Apart from programme costs from the project, data will be collected from the in-charges of the selected health units as to the inputs into their facilities. The cost evaluation data will be combined with the health outcome data to compute the cost-effectiveness analysis of the intervention.

Quantitative data (from the surveys) entered in netbooks will be synchronised with the master database in APHRC head office every day. Where data is missing or obvious inconsistencies are noted, the office editor will inform the data manager who will then contact the field teams for clarifications and, where need arises, send back queries to the field teams for completion of incomplete data or correction of the inconsistencies. Clean data will be exported for analysis to statistical software (STATA V.11.0; StataCorp LP, Texas, USA) for advanced cleaning and analysis. We will conduct basic tabulations and regression analyses comparing differences in the proportions of women in reproductive age and children under five years at baseline and end-line for variables such as contraceptive prevalence, vaccination coverage, skilled attended delivery, care-seeking for childhood illnesses, among others. These analyses will control for any differences in the samples (if any) at the two time points as well as the contribution of contextual factors that may have occurred in the course of the implementation.

Quantitative data from the health facilities, CHVs and the client satisfaction survey will be entered into Excel work books monthly. Basic analyses of the collated data will be conducted, including the methods of median, mean and range.

Qualitative data will be transcribed and saved in Word format. Transcribed Word files will be imported into NVIVO software (QSR International Pty Ltd) for coding and further analysis. Analysis across all transcripts will be conducted using a constant comparative method to identify themes and their repetitions and variations. The analysis will also aim to identify changes, if any, in various indicators, which could be attributed to the intervention.

\section{Project timeline and beneficiaries}

The project started in July 2012 and will last a total of 54 months (figure 5). The direct beneficiaries of the project are women of reproductive age and children under the age of 5 years in the two informal settlements who make up $20 \%$ and $14 \%$ of the population, respectively. ${ }^{12}$ In addition, five health facilities are being upgraded and the healthcare providers in the selected PNFP and other public and private health facilities are benefitting from training and skills upgrade. CHVs, the sCHMTs of the two subcounties where the study sites are 
located as well as the Nairobi County Health Management leadership are other direct beneficiaries. Residents of areas outside the NUDHSS as well as residents of the two slums who are male and/or older than 5 years but less than 15 years and/or older than 50 years are the indirect beneficiaries.

\section{ETHICS AND DISSEMINATION}

Ethical approval for the PAMANECH study was obtained from the Kenya Medical Research Institute. Study documents and consent forms detailing the scope of the project, activities and participant roles have been developed. All study participants and facilities in this research will be assigned unique study identifiers. The data set will be stored in a computerised database and study documentation and materials (including informed consent forms) will be stored in locked file cabinets when not in use at APHRC. Only the research team will have access to this information. Audio-taped interviews will also be labelled with the study identifier, rather than with names. The audio-taped interviews and informed consent forms will both be retained for a 2-year period, after which they will be destroyed. Data entry staff for this project will only have permission to retrieve/place materials with the authorisation of the investigators and under the condition that the staff members understand and have signed the confidentiality agreement.

Before presentation and or publication, participant and facility information will be de-identified. The findings will be summarised and packaged appropriately to facilitate uptake by policymakers. The findings will also be widely disseminated through workshops, conference presentations, newsletters, factsheets, internet blogs and academic publications.

\section{DISCUSSION}

The programme will demonstrate how access and utilisation of healthcare services by women of child-bearing age and children in the slums have improved as a result of the establishment of the six one-stop primary level centres. It is expected that the key outcomes in these groups will be reduced maternal and child deaths, lower incidence of childhood illnesses and improved maternal health. These changes will be brought about through the direct implementation of the interventions in the short term and indirectly by influencing government policy thereby ensuring sustainability of the programme in the long term. In addition, the programme will impact positively on the relationship between private and public providers in the districts, resulting in a common purpose and joint responsibility for improving the health of slum residents in the two districts. The programme will also strengthen the functioning of the community units in these settlements with improved supervision of CHVs and greater involvement of the communities in ensuring access to health services. The establishment of emergency transport systems and community security systems to support access to health services, especially at night, will provide learning opportunities for these and similar communities on effective approaches to reducing maternal and childhood morbidity and mortality caused by barriers to appropriate healthcare. One of the strengths of this project lies in linking learning with policy and action. It completes the triple helix of learning-policy-action. This project not only directly contributes to the wellbeing of slum dwellers, but will also facilitate interactions between all health sector players. Conventional projects are unidirectional; this project is multidirectional. It seeks to address most challenges in the healthcare system in a slum context: Infrastructural, human resource,

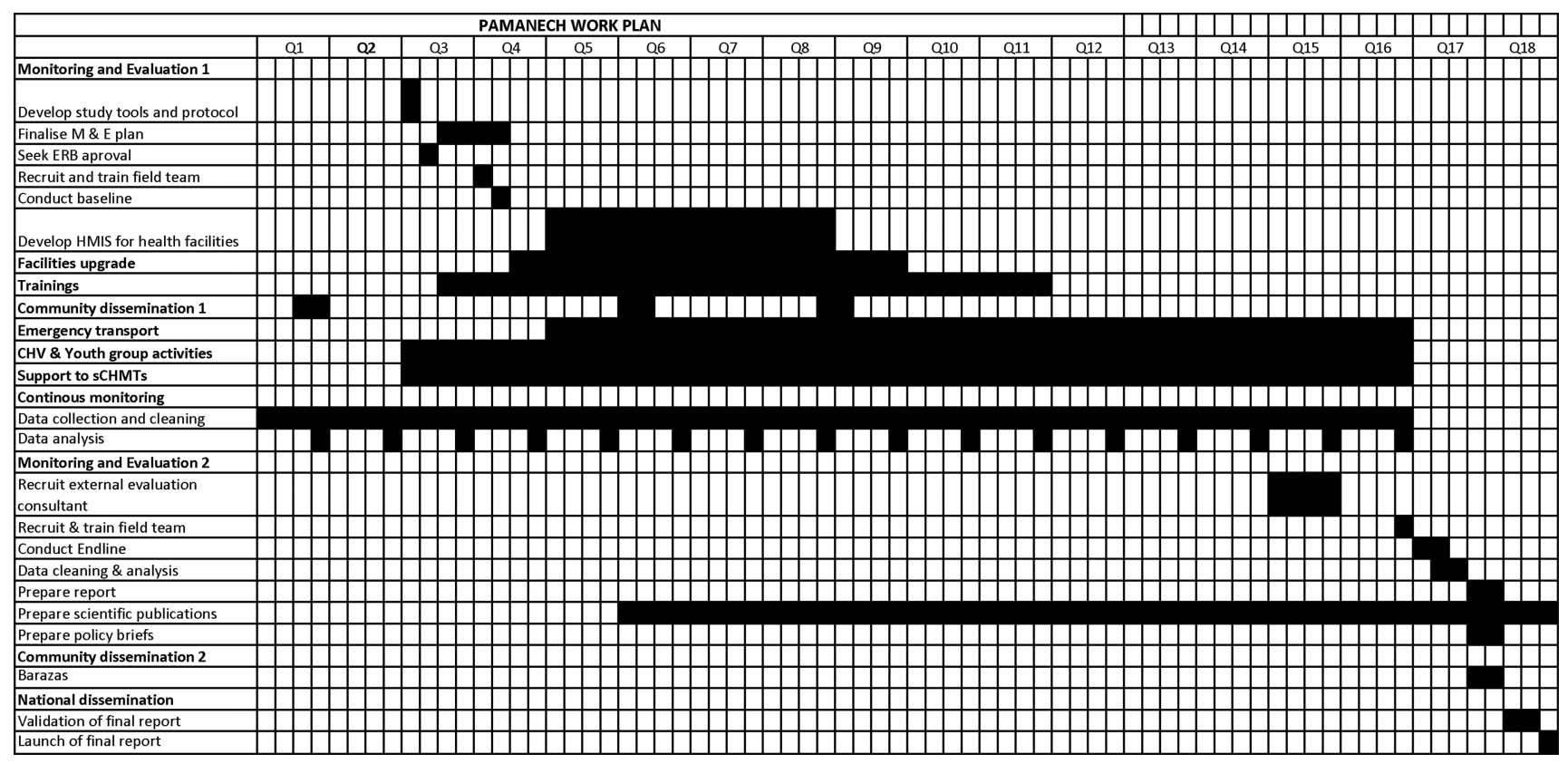

Figure 5 PAMANECH Project timeline. 
information, financing, technologies and supplies. It creates goodwill from policymakers and implementers. At the same time it demonstrates in action that intervention based on research evidence can change the health outcomes of a community. The initiative will bring together private healthcare providers, local health authorities, CHVs and community groups. It is, therefore, all encompassing and its success is necessarily tied to the extent and depth of interactions between all these groups. Through these interactions, all stakeholdersincluding the slum dwellers themselves-will play a role in creating the systemic change desired and will form long lasting relationships that could be used in scaling up the project. In addition, the inter-linkages with policymakers will catalyse a policy discourse and action on how to improve the health conditions of the urban poor.

One possible risk of the proposed intervention is interference with the health care market in the slums. By supporting a few credible providers, the remaining credible provider may lose clientele and be forced out of business. There are no guarantees that the supported providers will still be operating beyond the life of the project. To mitigate against this risk, we will extend some support to other credible providers such as supportive supervision from the sCHMTs, training sessions, improvements in HMIS systems and recommendations to MEDS for low-cost drug purchases. Finally, compliance by all actors is very important for the success of this project.

\section{CONCLUSION}

The study outlined in this protocol will assess the impact of strengthening of components of the healthcare systems on the MNCH services and population health outcomes in two informal settlements in Kenya. The study's findings will contribute to the body of knowledge on the effectiveness of public-private partnership in improving $\mathrm{MNCH}$ services and outcomes in urban informal settlements. Implementation of the protocol will generate evidence on the effectiveness, if any, of the intervention and possibly provide a model of publicprivate engagement for adoption by the local and central governments for under-served populations like slums in Kenya and other sub-Saharan African countries.

Acknowledgements We extend our sincere gratitude to the Viwandani and Korogocho communities for their continued support and participation in our research projects.

Contributors PB drafted the manuscript. CK, RE and AKZ conceived the project and its design, and participated in refining the manuscript. EK, JO and NN participated in refining the protocol. All authors read and approved the final manuscript.

Funding This project is supported by Comic Relief, UK, grant number GR002-12547. The funder had no role in the decision to write and publish this protocol. This work is also made possible through the generous core funding to APHRC by the William \& Flora Hewlett Foundation and the Swedish International Development Agency.
Competing interests None.

Ethics approval Kenya Medical Research Institute.

Provenance and peer review Not commissioned; internally peer reviewed.

Data sharing statement Additional information on the project can be obtained from Pauline Bakibinga: pbakibinga@aphrc.org.

Open Access This is an Open Access article distributed in accordance with the Creative Commons Attribution Non Commercial (CC BY-NC 4.0) license, which permits others to distribute, remix, adapt, build upon this work noncommercially, and license their derivative works on different terms, provided the original work is properly cited and the use is non-commercial. See: http:// creativecommons.org/licenses/by-nc/4.0/

\section{REFERENCES}

1. UNICEF. Information by country Kenya statistics. New York: UNICEF, 2010.

2. Kenya National Bureau of Statistics (KNBS). 2009 Population and housing census results Kenya. Kenya National Bureau of Statistics (KNBS). 2010

3. UN-HABITAT. The state of African cities 2010: governance, inequality and urban land markets. UN-HABITAT, 2010.

4. Ziraba AK, Mills S, Madise N, et al. The state of emergency obstetric care services in Nairobi informal settlements and environs: results from a maternity health facility survey. BMC Health Serv Res 2009;9:46

5. Bazant ES, Koenig MA, Fotso JC, et al. Women's use of private and government health facilities for childbirth in Nairobi's. Informal Settlements. Stud Fam Plann 2009;40:39-50.

6. UN-HABITAT. Sustainable urbanization: local action for urban poverty reduction, emphasis on finance and planning. UN-HABITAT, 2007.

7. Fotso JC, Mukiira C. Perceived quality of and access to care among poor urban women in Kenya and their utilization of delivery care: harnessing the potential of private clinics? Health Policy Plan 2012;27:505-15.

8. Kyobutungi C, Ziraba AK, Ezeh A, et al. The burden of disease profile of residents of Nairobi's slums: results from a demographic surveillance system. Popul Health Metr 2008;6:1.

9. Ekirapa A, Mgomella GS, Kyobutungi C. Civil society organizations: capacity to address the needs of the urban poor in Nairobi. J Public Health Pol 2012;33:404-22.

10. GOK. Draft public private partnership bill (February 2011). Nairobi: Treasury, 2011.

11. WHO. Everybody's business: strengthening health systems to improve health outcomes-WHO's framework for action. Geneva: World Health Organization, 2007.

12. Emina J, Beguy D, Zulu EM, et al. Monitoring of health and demographic outcomes in poor urban settlements: evidence from the Nairobi Urban Health and Demographic Surveillance System. J Urban Health 2011;88:200-18.

13. Ziraba AK, Madise N, Mills S, et al. Maternal mortality in the informal settlements of Nairobi city: what do we know. Reprod Health 2009;6:4755-6.

14. African Population and Health Research Center. Population and health dynamics in Nairobi's informal settlements. Nairobi: African Population and Health Research Center (APHRC), 2002.

15. African Population and Health Research Center. Population and health dymanics in Nairobi's informal settlements. Nairobi: African Population and Health Research Center (APHRC), 2014. Under preparation

16. NHIF. NHIF accreditation manual. 1st edn. Nairobi: NHIF, 2005

17. SafeCare basic health care standards.

18. Jahn A, De Brouwere V, eds. Referral in pregnancy and childbirth: concepts and strategies. In: Safe motherhood strategies: a review of the evidence Antwerp: ITG Press 2001.

19. Samai $O$, Sengeh $P$. Facilitating emergency obstetric care through transportation and communication, Bo, Sierra Leone. Int J Gynaecol Obstet 1997;59:S157-64.

20. Macintyre K, Hotchkiss DR. Referral revisited: community financing schemes and emergency transport in rural Africa. Soc Sci Medk 1999;49:1473-87. 